\title{
miR-1258: a novel microRNA that controls TMPRSS4 expression is associated with malignant progression of papillary thyroid carcinoma
}

\author{
Li-Juan Wang, Han-Qing Cai \\ Department of Endocrinology, The Second Hospital of Jilin University, Changchun, Jilin, China
}

\begin{abstract}
Introduction: MicroRNA-1258 (miR-1258) has been shown to play an anti-cancer role in a variety of cancers, but its relationship with papillary thyroid cancer (PTC) has not been reported. The emphasis of this research was to reveal the biological function of $m i R-1258$ in PTC and its potential mechanisms.

Material and methods: We measured miR-1258 expression in PTC cells and the transfection efficiency of miR-1258 mimic and miR-1258 inhibitor by quantitative real-time PCR (qRT-PCR) assay. Cell Counting Kit-8 assay (CCK8) and Transwell experiments were conducted to examine the influences of altering miR-1258 expression on the viability, migration, and invasion of PTC cells. Bioinformatics prediction and dual-luciferase experiment were performed to verify the target gene of miR-1258. Finally, we carried out a rescue assay to verify whether the regulation of miR-1258 on the biological behaviour of PTC cells needs to be achieved by regulating TMPRSS4.

Results: The outcomes revealed that miR-1258 was lowly expressed in PTC cell lines and miR-1258 showed the lowest expression in KTC1 and the highest expression in B-CPAP among all tested PTC cell lines. Overexpression of miR-1258 inhibited KTC-1 cell viability and ability to migrate and invade, whereas inhibition of miR-1258 in B-CPAP cells has the opposite effect. Furthermore, we affirmed that miR1258 can directly target TMPRSS4, and miR-1258 can reduce the biological malignant behaviour of PTC cells via regulation of TMPRSS4. Conclusion: Taken together, our research raised the possibility that miR-1258 was an anti-oncogene, which exerts its anti-cancer function by targeting TMPRSS4. Hence, it may be possible to treat PTC by targeting the miR-1258/TMPRSS4 axis in the future. (Endokrynol Pol 2020; 71 (2): 146-152)
\end{abstract}

Key words: miR-1258; TMPRSS4; target; PTC

\section{Introduction}

Papillary thyroid cancer (PTC), as the most common subtype of thyroid cancer, has the characteristics of high cure rate and long-term survival rate (> 95\%) [1-3]. In some patients with poor prognosis, recurrence and metastasis are the direct factors leading to death [4, $5]$. Although PTC is more common in female patients, the mortality rate of male patients is higher $[6,7]$. To date, most patients with PTC can be cured by surgery, but owing to the increasing incidence of PTC [8] and the high mortality rate of patients with recurrence and metastasis, a useful target of the diagnosis and therapy of PTC still need to be studied and developed.

MicroRNA (miRNA), as an endogenous small RNA in the non-coding region of the genome $[9,10]$, can regulate more than $30 \%$ of human genes and contributes to the regulation of various major diseases in humans [11]. Extensive research has reported the role of miRNAs in PTC; for example, up-regulation of miR-222 expression in PTC was associated with clinical risk stratification [12]. MiR-23a was down-regulated in PTC and participates in the regulation of PTC by targeting CCNG1 [13]. MiR-96-5p induced the invasion and migration of PTC cells by silencing the expression of CCDC67, thus accelerating the development of PTC [14]. In contrast, miR-506 can significantly reduce the migration and invasion ability of PTC cells [15]. MicroRNA-1258 (miR-1258), as one of the miRNAs, has been reported to participate in regulating the process of carcinoma and plays a tumour suppressive role in many cancers, such as NSCLC(17), ovarian cancer [18], osteosarcoma [19], oral squamous cell carcinoma [20], colorectal cancer [21], breast cancer [22], etc. Nevertheless, no previous research has been performed on the relationship between miR-1258 and PTC.

On the basis of existing literature data, we carried out studies in an effort to investigate the role of $m i R-1258$ in the viability, migration, and invasion of PTC cells, and to study its potential target genes. It was expected to provide a potentially useful strategy for the diagnosis and therapy of PTC. 


\section{Material and methods}

\section{Data collection}

In this study, we downloaded data from the The Cancer Genome Atlas (TCGA) database (https:// www.cancer.gov/about-nci/organization/ccg/research /structural-genomics/tcga) to analyse the difference of the expression of miR-1258 in PTC cells and normal cells. Similarly, the data of 510 tumour samples and 58 healthy controls were downloaded from the TCGA database and used to analyse differential expression of transmembrane protease serine 4 (TMPRSS4).

\section{Cell culture}

Human B-CPAP, BHT101, and KTC-1 PTC cell lines (Shanghai Cell Bank of Chinese Academy of Medical Sciences) and normal thyroid epithelial cell Nthy-ori 3-1 (European Collection of Cell Culture, Salisbury, UK) were routinely cultured in RPMI-1640 medium containing $10 \%$ FBS, $100 \mathrm{U} / \mathrm{ml}$ penicillin, and $0.1 \mathrm{mg} / \mathrm{ml}$ streptomycin at $37^{\circ} \mathrm{C}$ with $5 \% \mathrm{CO}_{2}$.

\section{Cell transfection}

MiR-1258 mimic/inhibitor and corresponding NC were obtained from Shanghai GenePharma Co., Ltd. (Shanghai, China), and pcDNA3.1-TMPRSS4, si-TMPRSS4, si-con were compounded by Shanghai GenePharma Co., Ltd. (Shanghai, China). The conventionally cultured cells were seeded in a six-well plate before transfection for $24 \mathrm{~h}$. When the cell fusion degree achieved $70-90 \%$, the culture medium was swapped to antibiotic-free and serum-free medium, and then transfected using Lipofectamine 2000 after $2 \mathrm{~h}$ The transfection was carried out according to the instructions of the Lipofectamine 2000 transfection kit. After 48 hours, the transfection was observed, and the cells were collected for further assays.

\section{Quantitative real-time PCR ( $q R T-P C R)$}

Total RNA was extracted by RNeasy Mini Kit (Qiage, Dusseldorf, Germany) according to the manufacturer's instructions. After that, M-MLV (Clontech, Palo Alto, USA) and miScript PCR reverse transcription kit (Qiagen, Dusseldorf, Germany) were used for reverse transcription. Then, using the qRT-PCR kit to test the expression level of miR-1258/TMPRSS4, GAPDH and U6 were used as the internal reference genes. The qRT-PCR reaction was carried out as follows: $95^{\circ} \mathrm{C}$ for $5 \mathrm{~min}, 95^{\circ} \mathrm{C}$ for $5 \mathrm{~s}$, and $60^{\circ} \mathrm{C}$ for $30 \mathrm{~s} ; 40$ cycles, using the $2{ }^{-\Delta \Delta C t}$ method to calculate the relative quantities. The specific primer sequences were:

MiR-1258:

F: 5'-GTTAGGATTAGGTCGTGG-3'

R: 5'-GAACATGTCTGCGTATCTC-3'

U6:

F: 5'-CTCGCTTCGGCAGCACA-3'

R: 5'-AACGCTTCACGAATTTGCGT-3'

TMPRSS4:

F: 5'-GACGAGGAGCACTGTGTCAAGA-3'

R: 5'-GAAACAGGCAGAGAACCAGTTCC-3'

GAPDH:

F: 5'-TAG ATGACACCCGTCCCTGA-3'

R: 5'-ACCTCCACCTGTCCTTAGTG-3'

\section{Western blotting assay}

Six-well plates with transfected cells were placed on ice, and protein was extracted by RIPA lysis buffer with protease inhibitor. Then a BCA protein quantitative kit was used to measure the protein content in the samples. About $20 \mu \mathrm{g}$ of the above protein was conducted to SDS-PAGE electrophoresis, followed by transferral of the separated protein sample to a PVDF membrane. After adding Western Sealing Solution (5\% skimmed milk powder) to seal for $1 \mathrm{~h}$, incubation of the membrane overnight at $4^{\circ} \mathrm{C}$ with primary antibody, followed by rinsing the incubated membrane three times with TBST for five minutes each time was carried out. Then the secondary antibody was added to the membrane and incubated for $1 \mathrm{~h}$ at room temperature. After rinsing with TBST for $3 \times 5 \mathrm{~min}$, the images were developed with an ECL Western blotting kit (Thermo Fisher Scientific, Inc.). Using Quantity One software to analyse the greyscale value of protein bands, GAPDH was used as the internal reference gene.

\section{Cell counting kit-8 (CCK8) assay}

The transfected cells were prepared into cell suspension and inoculated on a 96-well plate at a density of $1 \times 10^{5}$ cells/well, followed by conventional culturing in a $5 \% \mathrm{CO}_{2}$ incubator. OD values at 450 $\mathrm{nm}$ wavelength of each well were measured using a microplate reader at $0 \mathrm{~h}, 24 \mathrm{~h}, 48 \mathrm{~h}$, and $72 \mathrm{~h}$. Notably, $10 \mu \mathrm{l}$ of CCK8 solution was added to each well $2 \mathrm{~h}$ before detection.

\section{Transwell assay}

$40 \mu \mathrm{l}$ of Matrigel Matrix glue was added to the upper chamber of the Transwell chamber and incubated overnight at $37^{\circ} \mathrm{C}$ to make the Matrigel gelatinous. The migration assay did not require gluing treatment. After digestion, serum-free culture medium was used to prepare the cell suspension. The cell suspension was added to the upper chamber at the density of $1 \times 10^{5}$ cells, and $500 \mu \mathrm{l}$ of complete culture medium was added to the lower chamber. Then the chamber was cultured at $37^{\circ} \mathrm{C}$ for $24 \mathrm{~h}$ in a $5 \% \mathrm{CO}_{2}$ incubator. Afterwards, the chamber was removed and the remaining cells were wiped from the upper chamber with a cotton swab. The cells in the lower chamber were fixed with $4 \%$ paraformaldehyde for 15 minutes and stained with $0.1 \%$ crystal violet for three minutes. Finally, five visual fields were randomly selected under an optical microscope to observe and count the cells.

\section{Bioinformatics prediction}

The target gene of $m i R-1258$ was predicted by the target gene prediction and analysis websites miRbase (http://www.mirbase.org/) and TargetScan (http://www.targetscan.org/).

\section{Dual-luciferase reporter assay}

In order to verify whether miR-1258 can directly target TMPRSS4, dual-luciferase reporter assay was performed. The wild type and mutant type 3'UTR of TMPRSS4 were cloned into pmirGLO luciferase vector to construct a luciferase expression plasmid TMPRSS4-WT and TMPRSS4-MUT, respectively, and they were co-transfected into HEK-293T cells with miR-1258 mimic or miR-1258 mimic NC. Lipofectamine 2000 was used for transfection according to the instructions. Luciferase activity was detected after $24 \mathrm{~h}$.

\section{Statistical analysis}

The experimental data were analysed by SPSS22.0 statistical analysis software. T-test was used to compare the differences between the two groups, and one-way ANOVA analysis and post-test of Tukey were used to compare the differences between three or more groups. Statistical results were considered to be significantly different when $p<0.05$.

\section{Results}

\section{MiR-1258 was lowly expressed in PTC cell lines}

Firstly, the bioinformatics analysis results in Figure $1 \mathrm{~A}$ showed that miR-1258 expression was markedly lower in PTC tumour tissues than in normal tissues $(p<0.0001)$. Subsequently, we confirmed this difference in PTC cell lines by qRT-PCR assay. Figure 1B indicated that miR-1258 expression was markedly lower in PTC 
cell lines B-CPAP, BHT101, and KTC-1 than that in normal healthy cell Nthy-ori 3-1 ( $\mathrm{p}<0.01$ ). Interestingly, we also found that $m i R-1258$ showed unequal expression in different PTC cell lines, with the lowest expression in KTC-1 and the highest expression in B-CPAP.

\section{The effect of miR-1258 inhibitor/mimic transfection on the expression of miR-1258 in PTC cells}

To detect the function of miR-1258 inhibitor/mimic, we selected KTC-1 for over-expression assay and B-CPAP for knockdown assay. KTC-1 was transfected with miR-1258 mimic and miR-1258 mimic NC, simultaneously B-CPAP was transfected with miR-1258 inhibitor and miR-1258 inhibitor NC. Cells that were treated with transfection reagent were considered as controls. Then the expression of miR-1258 in KTC-1/ B-CPAP cells after transfection was detected by qRT-PCR. Figure 1CD shows that miR-1258 expression was markedly increased in KTC-1 cells transfected with miR-1258 mimic compared with that in KTC-1 cells transfected with miR-1258 mimic $\mathrm{NC}$ and in controls ( $\mathrm{p}<0.01$ ), while the expression of
miR-1258 was obviously reduced in CPAP cells transfected with miR-1258 inhibitor compared to that in B-CPAP cells transfected with miR-1258 inhibitor NC and in controls $(p<0.01)$. These results illustrated a successful transfection and a high transfection efficiency.

\section{The influence of altering the miR-1258 expression level on the biological behaviour of PTC cells}

To verify whether miR-1258 plays a biological function in PTC cells, CCK8 and Transwell experiments were set up to detect cell viability, migration, and invasion. It can be seen from Figure 2A that the cell viability of KTC-1 cells transfected with $m i R-1258$ mimic declined more than that in the control group $(\mathrm{p}<0.01)$, whereas it can be seen from Figure 2D that the cell viability showed a marked increase after inhibition of miR-1258 expression in B-CPAP cells ( $p<0.01$ ). The results obtained from the Transwell assay indicated that after overexpression of miR-1258 in KTC-1 cells, the number of migrating and invading cells through the Transwell membrane was markedly reduced compared to that in the control group (Fig. 2 BC, p $<0.01$ ), whereas

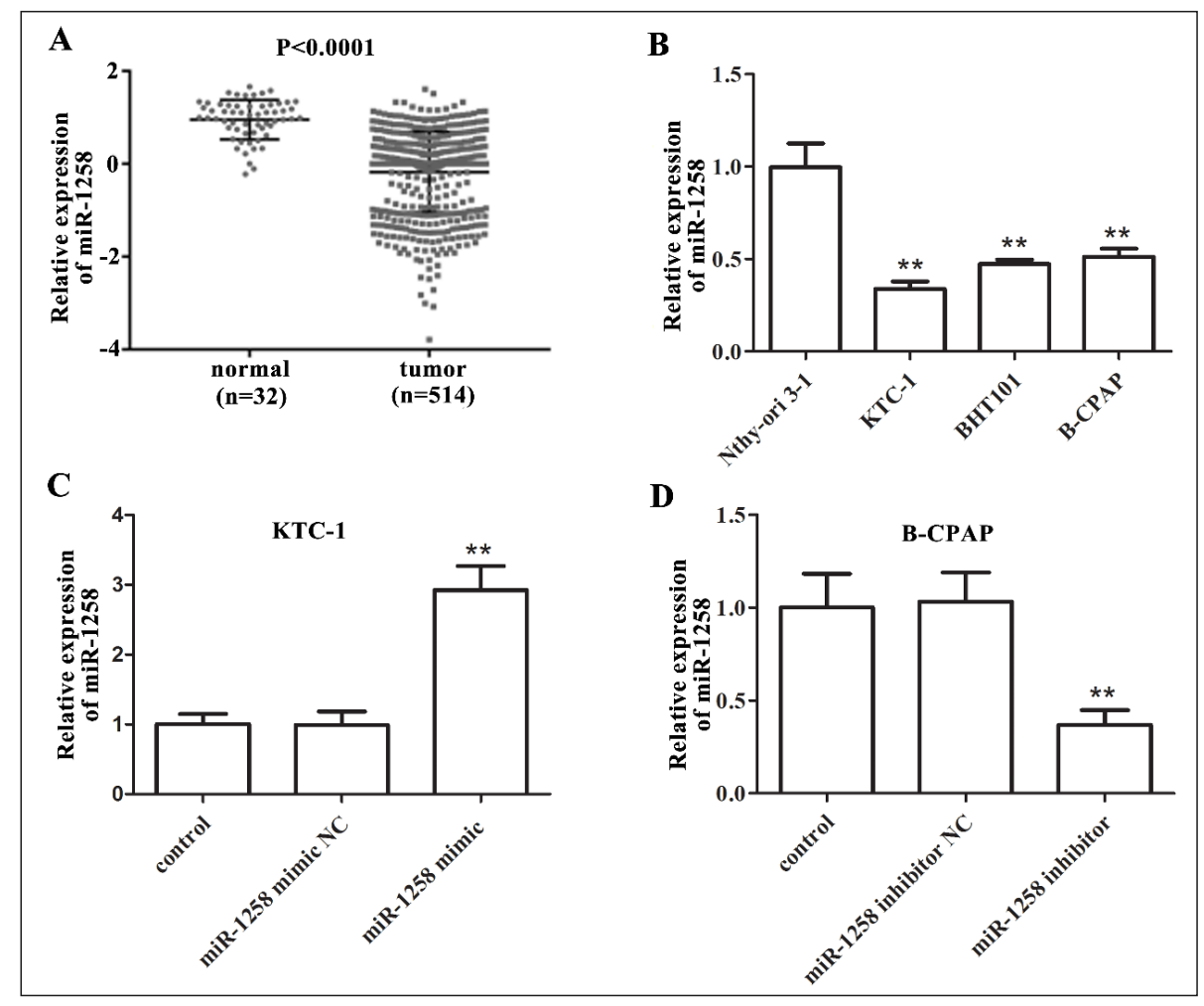

Figure 1. miRNA expression level of miR-1258 in papillary thyroid cancer (PTC). A. MiR-1258 was lowly expressed in the tumour group $(n=514)$ and the normal group $(n=59)$ on the basis of data from TCGA $(p<0.0001)$. B. Through quantitative real-time PCR ( $q R T-P C R)$ experiment, the expression of miR-1258 was markedly reduced in PTC cell lines B-CPAP, BHT101, KTC-1 than that in normal healthy cell Nthy-ori 3-1 $\left.{ }^{* *} p<0.01\right)$. C. The expression of miR-1258 in KTC-1 cells transfected with miR-1258 mimic showed an obvious increase compared with that in KTC-1 cells transfected with mimic NC and that in controls $\left({ }^{* *} p<0.01\right)$. D. The expression of miR-1258 in B-CPAP cells transfected with miR-1258 inhibitor was significantly decreased compared with that in B-CPAP cells transfected with miR-1258 inhibitor NC and in controls $\left({ }^{* *} p<0.01\right)$ 


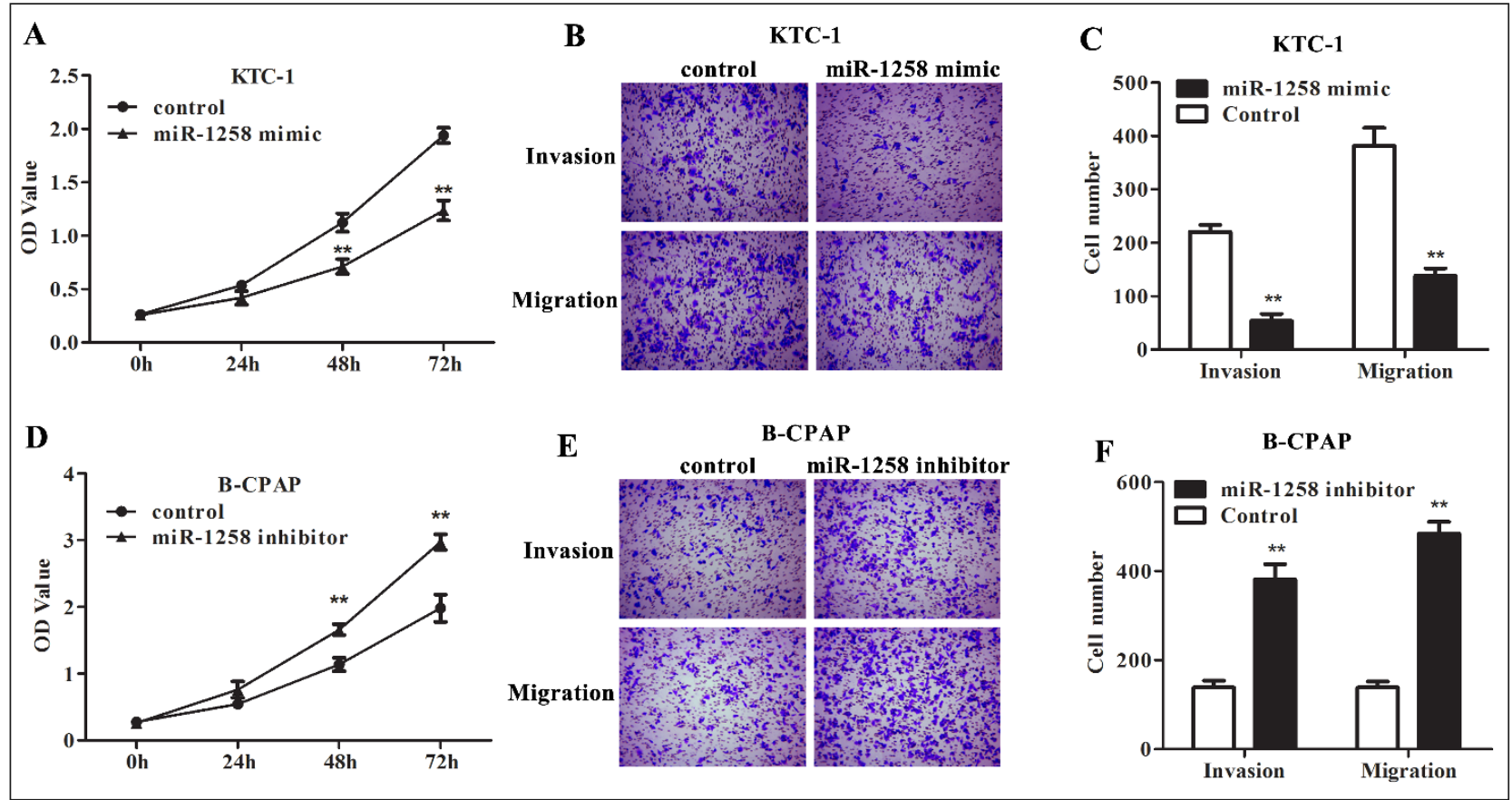

Figure 2. Influence of altering the expression of miR-1258 on the cell biological behaviour in KTC-1 and B-CPAP. A. Cell Counting Kit-8 (CCK8) assay results indicated that up-regulation of miR-1258 expression markedly declined the viability of KTC-1 cells compared to that in controls $\left({ }^{* *} p<0.01\right)$. B. Down-regulation of miR-1258 expression markedly enhanced the viability of B-CPAP cells compared to that in controls $\left({ }^{* *} p<0.01\right)$. C, D. Transwell assay results illustrated that up-regulation of miR-1258 obviously reduced the number of migrating and invading cells in KTC-1 compared to that in controls $\left.{ }^{* *} p<0.01\right)$. E, F. Inhibition of miR-1258 expression can improve the cell invasion and metastasis of B-CPAP cells compared to that in controls $\left({ }^{* *} p<0.01\right)$

suppression of miR-1258 in B-CPAP cells showed the opposite effect (Fig. 2EF, p < 0.01). Overall, these results demonstrate that overexpression of $m i R-1258$ can inhibit the cell viability, migration, and invasion in PTC, and thus miR-1258 may be a tumour suppressor gene in PTC.

\section{MiR-1258 directly targets TMPRSS4}

To explore the mechanism of regulation of PTC by miR-1258 we used a target gene prediction website to predict and analyse the target of $m i R-1258$. Bioinformatics analysis indicated that TMPRSS4 was more highly expressed in PTC cells than in normal cells (Fig. 3A, p < 0.0001), and it may be a potential target gene for $m i R-1258$, which has predicted target sites for $m i R-1258$ in the $3^{\prime}$-UTR region (Fig. 3B). To validate further whether TMPRSS4 is a direct target gene of $m i R-1258$, TMPRSS4-WT or TMPRSS4-MUT and $m i R-1258$ mimic or $m i R-1258$ mimic NC were co-transfected into HEK 293T cells to determine the cell luciferase activity. From Figure 3C we can see that miR-1258 mimic significantly reduced the luciferase activity of wild-type compared to the negative control group ( $\mathrm{p}<0.01$ ), whereas the luciferase activity of the mutant did not change markedly compared with the control group.
Subsequently, Western blot assay was carried out to test the influence of overexpression/suppression of $m i R-1258$ on TMPRSS4 protein levels. The results from Figure 3DE revealed that the level of TMPRSS4 protein in KCT-1 cells transfected with $m i R-1258$ mimic decreased significantly compared to that in the control group ( $\mathrm{p}<0.01$ ), while the level of TMPRSS4 was markedly higher in KCT-1 cells co-transfected with mimic and pcDNA3.1-TMPRSS4 compared to that in the $m i R-1258$ mimic group ( $\mathrm{p}<0.01$ ). Simultaneously, it can be concluded from Figure 3EF that inhibition of $m i R-1258$ expression in B-CPAP cells can significantly increase the level of TMPRSS4 protein compared to the control group ( $\mathrm{p}<0.01$ ), whereas TMPRSS4 protein level in B-CPAP cells co-transfected with miR-1258 inhibitor and si-TMPRSS4 was reduced significantly compared to those in the inhibitor group. Overall, these results illustrate that $m i R-1258$ can directly target the TMPRSS4 gene and negatively regulate its expression.

\section{Regulation of the miR-1258/TMPRSS4 axis on the phenotype of PTC}

To confirm whether $m i R-1258$ regulates the biological behaviour of PTC cells by regulating TMPRSS4, a rescue experiment was performed. Firstly, KTC-1 cells were 


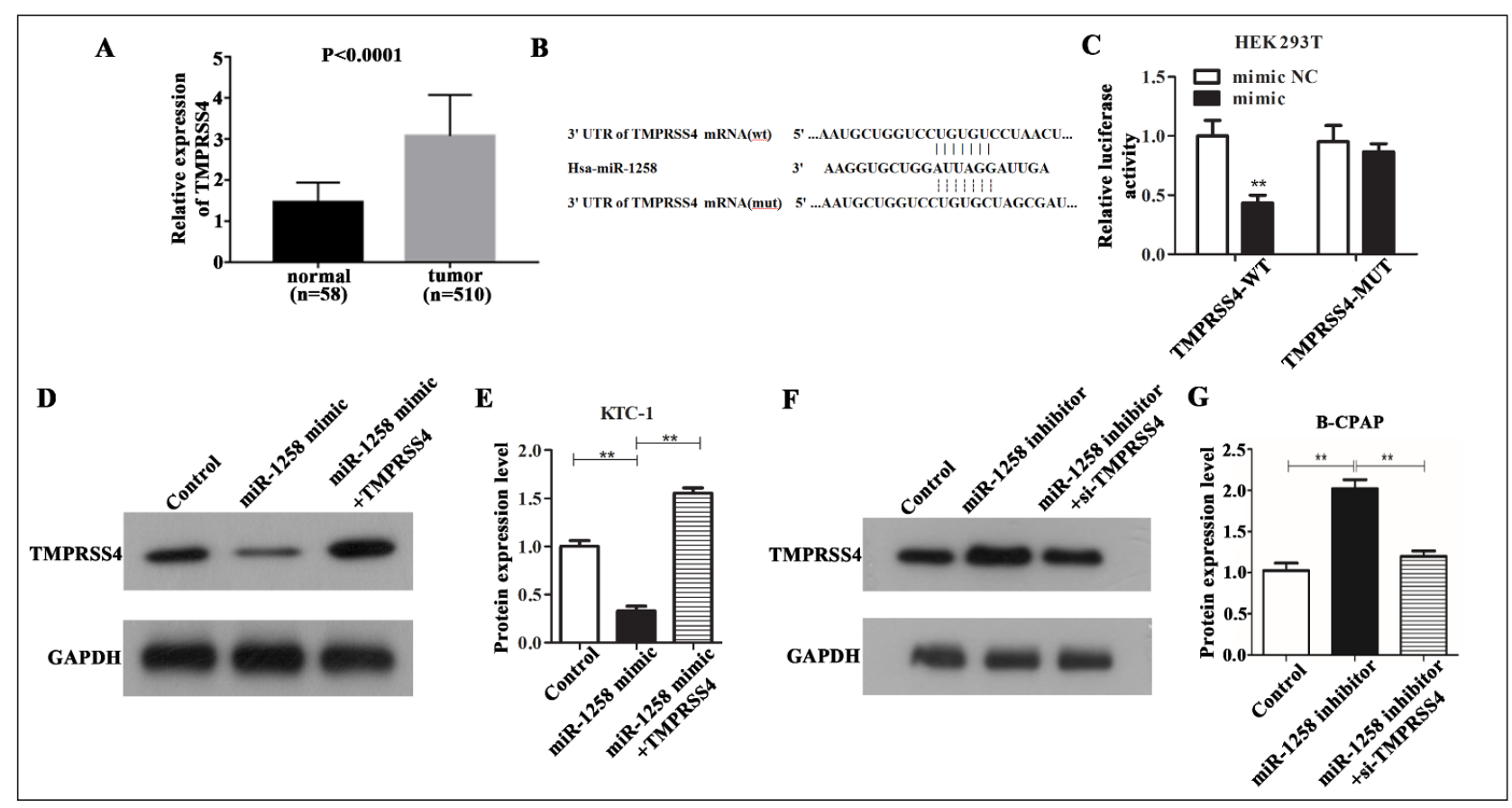

Figure 3. MiR-1258 directly targets TMPRSS4. A. Relative expression level of TMPRSS4 in normal cells $(n=58)$ and tumour cells $(n=510)$ was analysed on the basis of data from TCGA $(p<0.0001)$. B. Alignment of miR-1258 with TMPRSS4 sequences. C. The fluorescence intensity was markedly decreased in HEK 293 T cells co-transfected with TMPRSS4-WT and miR-1258 mimic $(p<0.01)$, while the fluorescence intensity of TMPRSS4-MUT and miR-1258 mimic co-transfected cells was almost unchanged. D, E. Overexpression of miR-1258 markedly decreased the level of TMPRSS4 protein compared with controls in KTC-1 cells $\left({ }^{* *} p<0.01\right)$, while the TMPRSS4 protein level was markedly increased in pcDNA 3.1-HAO2 and miR-1258 mimic co-transfected cells compared to the miR-1293 mimic group $\left.{ }^{* *} p<0.01\right)$. F, G. Inhibition of miR-1258 in B-CPAP cell can elevate the expression of TMPRSS4 $\left({ }^{* *} p<0.01\right)$, whereas knockdown of TMPRSS4 in the miR-1258 inhibitor group, TMPRSS4 protein level was markedly lower compared with that in the miR-1258 inhibitor group $\left({ }^{* *} p<0.01\right)$

transfected with miR-1258 mimic or miR-1258 mimic plus pcDNA3.1-TMPRSS4, and B-CPAP cells were transfected with miR-1258 inhibitor or miR-1258 inhibitor plus si-TMPRSS4, and negative control groups were set respectively. The viability, migration, and invasion of cells under different conditions were examined by CCK8 and Transwell experiments. As shown in Figure $4 \mathrm{~A}-\mathrm{C}$, the cell viability, migration, and invasion were reduced significantly more in KTC- 1 cells transfected with miR-1258 mimic than that in the controls $(\mathrm{p}<0.01)$, whereas after overexpression of TMPRSS4 in the miR-1258 mimic group, the cell viability and the number of migrating and invading cells were markedly elevated compared to those in the mimic group $(\mathrm{p}<0.01)$. At the same time, down-regulation of miR-1258 expression in B-CPAP cells significantly increased cell viability, migration, and invasive ability, while knockdown of TMPRSS4 in the inhibitor group showed the opposite effect. This suggests that overexpression of TMPRSS4 can reverse the anti-cancer properties of miR-1258. Thence, miR-1258 can reduce the viability, migration, and invasion of PTC cells via regulating TMPRSS4.

\section{Discussion}

In this paper, we found for the first time that miR-1258 was lowly expressed in PTC cell lines. Subsequently, a series of experimental results showed that suppression of miR-1258 in PTC can improve cell viability, migration, and invasion, while overexpression of miR-1258 showed the opposite effect. Finally, we confirmed that miR-1258 could directly target TMPRSS4, and it promoted the biological behaviour of PTC cells via regulation of TMPRSS4.

To date, a number of studies have revealed that miR-1258 has an imbalanced expression in various cancers. For example, down-regulated miR-1258 expression in osteosarcoma was related to unfavourable prognosis [19]. MiR-1258, as an anti-cancer gene, also plays a role in inhibiting the process of oral squamous cell carcinoma [20]. The anti-cancer effect of miR-1258 in colorectal cancer was achieved by negative regulation of E2F8 [21]. MiR-1258 was a diagnostic and therapeutic marker of hepatocellular carcinoma [23], and also can be used as a downstream functional target to regulate the process of hepatocellular carcinoma 


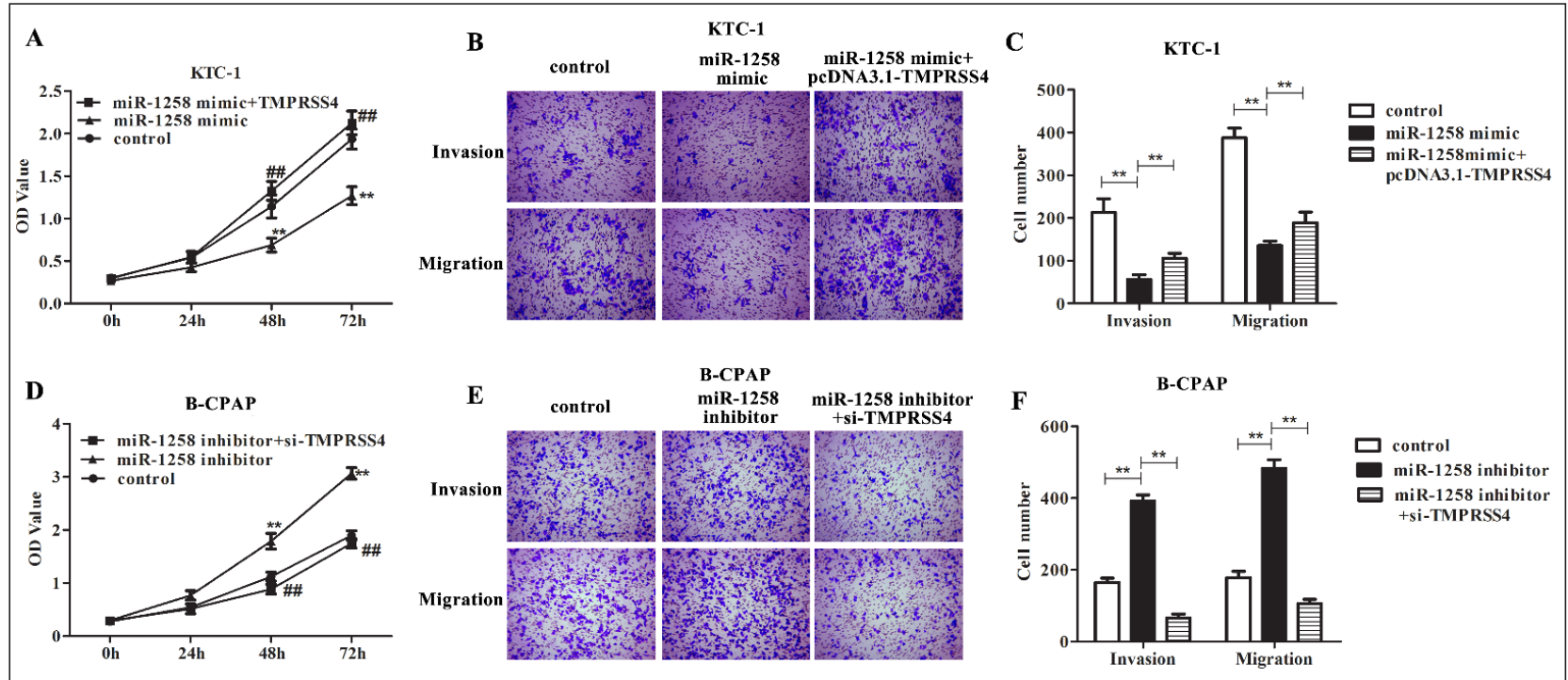

Figure 4. The effect of the miR-1258/TMPRSS4 axis on the function of PTC. A-C. Overexpression of miR-1258 significantly reduced the viability, invasion, and metastasis of KTC-1 cells compared to that in the control group $\left({ }^{* *} p<0.01,{ }^{\# \#} p<0.01\right)$, while the biological behaviour of KTC-1 cells co-transfected with pcDNA3.1-TMPRSS4 and miR-1258 mimic was markedly elevated compared to that in the miR-1293 mimic group. $\left({ }^{* *} p<0.01,{ }^{* \#} p<0.01\right)$. D-F. Suppression of miR-1293 markedly induced the viability, invasion, and metastasis of B-CPAP cells compared to those in the control group $\left.{ }^{* *} p<0.01\right)$, while the biological behaviour of B-CPAP cells co-transfected with si-TMPRSS4 and miR-1293 inhibitors was significant reduced compared to that in the miR-1293 inhibitor group $\left({ }^{*} p<0.01\right)$

[24]. Recently, Filippova EA et al. found that methylation of $m i R-1258$ can be used as a predictor of ovarian cancer metastasis [18]. This paper first identified the low expression of MIR-1258 in PTC, and up-regulation of miR-1258 markedly reduced the viability, migration, and invasion of PTC cells, while suppression of miR-1258 showed the opposite effect. Taken together, we concluded that $m i R-1258$ may has the tumour suppressor function of regulating the biological behaviour of PTC cells.

In this paper, we identified that TMPRSS 4 might be a target gene of miR-1258 by using a target gene prediction website. Type II transmembrane serine proteases (TTSP) have been found to be dysfunctional in a variety of cancers, which can activate signalling pathways leading to cancer [25]. TMPRSS4, as a new member of TTSP, has been found to be highly expressed in many cancers, such as pancreatic cancer [26], gastric cancer [27], lung adenocarcinoma [28], hepatocellular carcinoma [29], and breast cancer [30]. In addition, Mahati et al. pointed out that up-regulation of TMPRSS4 expression can promote the occurrence and development of various biological behaviours of cancer cells [29]. Based on the bioinformatics analysis and experimental results, we verified that TMPRSS4 was the direct target gene of $m i R-1258$, and up-regulation of TMPRSS4 expression reduced the influence of $m i R-1258$ to inhibit the viability, migration, and invasion in PTC cells, while knockdown of TMPRSS4 has the opposite effect. Hence, it is possible that $m i R-1258$ plays an anti-cancer role by regulating
TMPRSS4 in PTC, but the underlying mechanisms require further study.

From this paper, we concluded that miR-1258 was lowly expressed in PTC, and overexpression of miR-1258 can inhibit cell viability, migration, and invasion, which raises the standpoint that $m i R-1258$ is an anti-oncogene. Furthermore, we found that $m i R-1258$ may play an anti-cancer role in PTC by regulating TMPRSS4, suggesting that the miR-1258/TMPRSS4 axis may be a potential biomarker and therapeutic target for the diagnosis and therapy of PTC. In the next step, we will further validate our experimental data through in vivo experiments.

\section{References}

1. Miccoli P, Bakkar S. Surgical management of papillary thyroid carcinoma: an overview. Updates Surg. 2017; 69(2): 145-150, doi: 10.1007/s13304-017-0449-5, indexed in Pubmed: 28405952.

2. Pusztaszeri M, Auger M. Update on the cytologic features of papillary thyroid carcinoma variants. Diagn Cytopathol. 2017; 45(8): 714-730, doi: 10.1002/dc.23703, indexed in Pubmed: 28262004.

3. Wang Y, Han J, Lv Y, et al. miR-29a inhibits proliferation, invasion, and migration of papillary thyroid cancer by targeting DPP4. Onco Targets Ther. 2019; Volume 12: 4225-4233, doi: 10.2147/ott.s201532, indexed in Pubmed: 31213841.

4. Fagin JA, Wells SA. Biologic and Clinical Perspectives on Thyroid Cancer N Engl J Med. 2016; 375(23): 2307, doi: 10.1056/NEJMc1613118, indexed in Pubmed: 27959677.

5. Xing M, Alzahrani AS, Carson KA, et al. Association between BRAF V600E mutation and recurrence of papillary thyroid cancer. J Clin Oncol. 2015 ; 1(33): 42-50, doi: 10.1200/JCO.2014.56.8253, indexed in Pubmed: 25332244.

6. Zang C, Sun J, Liu W, et al. miRNA-21 promotes cell proliferation and invasion via VHL/PI3K/AKT in papillary thyroid carcinoma. Hum Cell. 2019; 32(4): 428-436, doi: 10.1007/s13577-019-00254-4, indexed in Pubmed: 31161410

7. Kilfoy BA, Devesa SS, Ward MH, et al. Gender is an Age-Specific Effect Modifier for Papillary Cancers of the Thyroid Gland. Cancer Epide- 
miol Biomarkers Prev. 2009; 18(4): 1092-1100, doi: 10.1158/1055-9965. epi-08-0976, indexed in Pubmed: 19293311.

8. Zhang W, Sun W, Qin Y, et al. Knockdown of KDM1A suppresses tumour migration and invasion by epigenetically regulating the TIMP1/MMP9 pathway in papillary thyroid cancer. J Cell Mol Med. 2019; 23(8): 4933-4944, doi: 10.1111/jcmm.14311, indexed in Pubmed: 31211500.

9. Rupaimoole R, Slack FJ. MicroRNA therapeutics: towards a new era for the management of cancer and other diseases. Nat Rev Drug Discov. 2017; 16(3): 203-222, doi: 10.1038/nrd.2016.246, indexed in Pubmed: 28209991.

10. Setoyama T, Ling H, Natsugoe S, et al. Non-coding RNAs for medical practice in oncology. Keio J Med. 2011; 60(4): 106-113, doi: 10.2302/kjm.60.106, indexed in Pubmed: 22200634

11. Chan B, Manley J, Lee J, et al. The emerging roles of microRNAs in cancer metabolism. Cancer Lett. 2015; 356(2 Pt A): 301-8, doi: 10.1016/j. canlet.2014.10.011, indexed in Pubmed: 25451319.

12. Xiang D, Tian B, Yang T, et al. miR-222 expression is correlated with the ATA risk stratifications in papillary thyroid carcinomas. Medicine (Baltimore). 2019; 98(25): e16050, doi: 10.1097/MD.0000000000016050, indexed in Pubmed: 31232941.

13. Yin JJ, Cheng XY. MicroRNA-23a inhibits the growth of papillary thyroid carcinoma via regulating cyclin G1. Eur Rev Med Pharmacol Sci. 2019; 23(8): 3431-3439, doi: doi: 10.26355/eurrev_201904_17707, indexed in Pubmed: 31081097.

14. Liu ZM, Wu ZY, Li WH, et al. MiR-96-5p promotes the proliferation, invasion and metastasis of papillary thyroid carcinoma through down-regulating CCDC67. Eur Rev Med Pharmacol Sci. 2019; 23(8): 3421-3430, doi: 10.26355/eurrev_201904_17706, indexed in Pubmed: 31081096.

15. Zhu J, Zhang Q, Jin XY, et al. MiR-506 suppresses papillary thyroid carcinoma cell proliferation and metastasis via targeting IL17RD. Eur Rev Med Pharmacol Sci. 2019; 23(7): 2856-2862, doi: 10.26355/eurrev_201904_17563, indexed in Pubmed: 31002136.

16. Liu F, Yin $\bar{R}$, Chen $X$, et al. Over-expression of miR-206 decreases the Euthyrox-resistance by targeting MAP4K3 in papillary thyroid carcinoma. Biomed Pharmacother. 2019; 114: 108605, doi: 10.1016/j. biopha.2019.108605, indexed in Pubmed: 30904818.

17. Jiang W, Wei Ke, Pan C, et al. MicroRNA-1258 suppresses tumour progression via GRB2/Ras/Erk pathway in non-small-cell lung cancer. Cell Prolif. 2018; 51(6): e12502, doi: 10.1111/cpr.12502, indexed in Pubmed: 30069987.

18. Filippova EA, Loginov VI, Burdennyi AM, et al. Hypermethylated Genes of MicroRNA in Ovarian Carcinoma: Metastasis Prediction Marker Systems. Bull Exp Biol Med. 2019; 167(1): 79-83, doi: 10.1007/s10517-019-04465-5, indexed in Pubmed: 31177462.

19. Liu W, Zhou Z, Zhang Qi, et al. Overexpression of miR-1258 inhibits cell proliferation by targeting AKT3 in osteosarcoma. Biochem Biophys Res
Commun. 2019; 510(3): 479-486, doi: 10.1016/j.bbrc.2019.01.139, indexed in Pubmed: 30737029.

20. Zhang H, Jiang S, Guo L, et al. MicroRNA-1258, regulated by c-Myb, inhibits growth and epithelial-to-mesenchymal transition phenotype via targeting SP1 in oral squamous cell carcinoma. J Cell Mol Med. 2019; 23(4): 2813-2821, doi: 10.1111/jcmm.14189, indexed in Pubmed: 30734471.

21. Zhang Z, Li J, Huang Y, et al. Upregulated miR-1258 regulates cell cycle and inhibits cell proliferation by directly targeting E2F8 in CRC. Cell Prolif. 2018; 51(6): e12505, doi: 10.1111/cpr.12505, indexed in Pubmed: 30144184

22. Shi J, Chen P, Sun J, et al. MicroRNA-1258: An invasion and metastasis regulator that targets heparanase in gastric cancer. Oncol Lett. 2017; 13(5): 3739-3745, doi: 10.3892/ol.2017.5886, indexed in Pubmed: 28521475.

23. Hu M, Wang M, Lu H, et al. Loss of miR- 1258 contributes to carcinogenesis and progression of liver cancer through targeting CDC28 protein kinase regulatory subunit 1B. Oncotarget. 2016; 7(28): 43419-43431, doi: 10.18632/oncotarget.9728, indexed in Pubmed: 27270326.

24. Zou H, Xu X, Luo L, et al. Hsa_circ_0101432 promotes the development of hepatocellular carcinoma ( $\overline{\mathrm{H}} \mathrm{CC}) \bar{b}$ by adsorbing miR-1258 and miR-622. Cell Cycle. 2019; 18(19): 2398-2413, doi: 10.1080/15384101.2019.1618120 , indexed in Pubmed: 31095447.

25. Tanabe LM, List K. The role of type II transmembrane serine protease-mediated signaling in cancer. FEBS J. 2017; 284(10): 1421-1436, doi: 10.1111/febs.13971, indexed in Pubmed: 27870503.

26. Cheng Y, Wang K, Geng L, et al. Identification of candidate diagnostic and prognostic biomarkers for pancreatic carcinoma. EBioMedicine. 2019; 40: 382-393, doi: 10.1016/j.ebiom.2019.01.003, indexed in Pubmed: 30639415.

27. Yuan H, Chen Z, Bai S, et al. Molecular mechanisms of lncRNA SMARCC2/miR-551b-3p/TMPRSS4 axis in gastric cancer. Cancer Lett. 2018; 418: 84-96, doi: 10.1016/j.canlet.2018.01.032, indexed in Pubmed: 29337109.

28. Fan X, Liang Y, Liu Y, et al. The upregulation of TMPRSS4, partly ascribed to the downregulation of miR-125a-5p, promotes the growth of human lung adenocarcinoma via the NF- $\kappa$ B signaling pathway. Int J Oncol. 2018; 53(1): 148-158, doi: 10.3892/ijo.2018.4396, indexed in Pubmed: 29750426.

29. Mahati S, Bolati D, Yang Y, et al. TMPRSS4 promotes cancer stem cell traits by regulating CLDN1 in hepatocellular carcinoma. Biochem Biophys Res Commun. 2017; 490(3): 906-912, doi: 10.1016/j.bbrc.2017.06.139, indexed in Pubmed: 28651932.

30. Li XM, Liu WL, Chen Xu, et al. Overexpression of TMPRSS4 promotes tumor proliferation and aggressiveness in breast cancer. Int J Mol Med. 2017; 39(4): 927-935, doi: 10.3892/ijmm.2017.2893, indexed in Pubmed: 28259959. 